\title{
Cardiovascular Effects of Long-Term Vitamin D Supplementation: Summarised by Many but Studied by Few
}

\author{
Mark Butlin Alberto P. Avolio \\ Department of Biomedical Sciences, Faculty of Medicine and Health Sciences, Macquarie \\ University, Sydney, NSW, Australia
}

Vitamin D supplementation has been primarily studied for its effect on mineral metabolism and, therefore, bone resorption. Vitamin D is also known to play a role in the reninangiotensin-aldosterone system [1] and in inflammation [2] and is produced by vascular endothelial cells [3]. It is, therefore, a strong hypothesis that plasma vitamin D levels influence cardiovascular factors in terms of blood pressure, vascular function and cardiovascular risk. In this issue of Pulse, Veloudi et al. [4] provide a qualitative review of a selection of randomised controlled trials (RCTs) concerning the effect of vitamin D supplementation on blood pressure and measures of arterial stiffness. They also include a descriptive review of some studies concerning cardiovascular outcomes of low serum levels of vitamin D. The article presents a graphical representation of sample size and study duration of 36 studies investigating peripheral blood pressure and 9 studies investigating arterial stiffness. In so doing, the article highlights that very few of these studies show a beneficial effect of vitamin D supplementation. The graphical presentation of the data also allows a visual representation of the previous finding, demonstrated statistically, that there is no relationship between an increased dose of vitamin D supplement and beneficial cardiovascular effect [5].

The effect of vitamin D supplementation on cardiovascular factors is of great clinical interest, demonstrated by the number of studies identified in meta-analyses in recent years. This year alone, quantitative meta-analyses of arterial stiffness outcomes have been published by Upala et al. [6] and Rodríguez et al. [7] summarising 7 and 18 RCTs, respectively. A recent quantitative meta-analysis on peripheral blood pressure outcomes identified 46 independent vitamin D supplementation trials [5]. These quantitative studies, although not included in the review by Veloudi et al. [4], support the same conclusion which Veloudi et al. [4] make from a qualitative review of RCTs, namely that vitamin D supplementation has no effect on arterial stiffness or blood pressure. However, Rodríguez et al. [7] surmise that larger, well-designed 
Butlin and Avolio: Cardiovascular Effects of Long-Term Vitamin D Supplementation: Summarised by Many but Studied by Few

RCTs are required to make sound conclusions on the effect of vitamin D supplementation on arterial stiffness. In terms of endothelial function, a quantitative meta-analysis published this year identified 16 RCTs [8], the summary of which also showed no effect of vitamin D supplementation.

Such is the level of research into the field that in 2014, Theodoratou et al. [9] conducted a review of 107 systematic reviews and 74 meta-analyses on plasma vitamin D levels and vitamin D supplementation across multiple health outcomes. Here, too, the authors concluded that the current literature provides no strong evidence for beneficial effects of vitamin D supplementation. However, they again raise the limitation of a commonly short study duration and also highlight that many studies do not measure plasma vitamin D concentration, not permitting for control of vitamin D levels given that the absorption and metabolism differs between individuals.

In terms of cardiovascular events, a meta-analysis of 18 RCTs showed plausible protection against cardiac failure in older people [10], but only with inclusion of patients with a high-risk profile of cardiovascular events. Previous studies did not show any effect on cardiovascular end points of vitamin D supplementation (51 studies) [11]. One meta-analysis of 18 RCTs identified a reduction in all-cause mortality with increased vitamin D [12], though the included studies relied heavily on high-risk groups with osteoporosis and analysed both plasma vitamin D levels and supplementary vitamin D, and no individual study identified a positive benefit of vitamin D for mortality [13]. This lack of statistical power is consistent with a metaanalysis of 8 studies on cardiovascular disease mortality, where a trend towards reduced mortality could not be shown to be statistically significant across the analysis [14]. A more recent meta-analysis found that the effect of vitamin D supplementation on all-cause mortality was only seen for vitamin D administration of 3 years or more [15]. Of the studies identified by Veloudi et al. [4], only 2 had a duration of 3 years or more, and this may present a major limitation in the available data on the investigation of the effect of vitamin D supplementation on blood pressure and arterial stiffness. However, Veloudi et al. [4] did attempt to address the duration of studies within the limitations of the data available by describing studies above and below a 6-month duration, with the majority of studies falling into vitamin D supplementation of less than 6 months. This supports the conclusion of Rodríguez et al. [7] that betterdesigned (and longer-duration) studies may be needed to discover any effect of vitamin D supplementation.

On the weight of the many quantitative meta-analyses of the effect of vitamin D supplementation on cardiovascular factors and end points, it can be concluded that vitamin D supplementation has no beneficial effect, and the review by Veloudi et al. [4] provides a graphical and qualitative summary of some of this evidence. Despite the number of studies on vitamin D supplementation and the number of quantitative meta-analyses of these data, it is repeatedly highlighted that the majority of studies follow a short duration that is unlikely to allow cardiovascular benefit and often do not control for circulating levels of vitamin D or vitamin D deficiency.

At this stage, the evidence indicates that short-term vitamin D supplementation imparts no cardiovascular benefit. In terms of longer-term administration of vitamin D, we are reliant on only a few studies in postmenopausal women [16], healthy elderly men [17] and normotensive men [18], all of which were studies that included combined calcium and vitamin D supplementation and were designed to investigate bone loss, not cardiovascular factors. Cardiovascular events have been examined in follow-up studies of 1-6 years' duration, again with the primary outcome in all studies being non-cardiovascular related [10]. Ignoring the limitations of these studies in investigating cardiovascular factors and end points, these too generally indicate no beneficial effect of vitamin D supplementation. Without a specifically designed long-term study on cardiovascular effects of vitamin D supplementation, it is difficult 
Butlin and Avolio: Cardiovascular Effects of Long-Term Vitamin D Supplementation:

Summarised by Many but Studied by Few

to state with confidence that vitamin D supplementation has no cardiovascular benefit. However, given the overwhelming incidental evidence against a cardiovascular benefit of vitamin D supplementation, it is unlikely that such a costly and time-consuming study will be conducted.

\section{Disclosure Statement}

The authors have no financial conflicts of interest that are related to this article.

\section{References}

1 Li YC, Qiao G, Uskokovic M, Xiang W, Zheng W, Kong J: Vitamin D: a negative endocrine regulator of the reninangiotensin system and blood pressure. J Steroid Biochem Mol Biol 2004;89-90:387-392.

2 Almerighi C, Sinistro A, Cavazza A, Ciaprini C, Rocchi G, Bergamini A: 1 $\alpha, 25$-Dihydroxyvitamin D3 inhibits CD40L-induced pro-inflammatory and immunomodulatory activity in human monocytes. Cytokine 2009; 45: 190-197.

3 Zehnder D, Bland R, Chana RS, Wheeler DC, Howie AJ, Williams MC, et al: Synthesis of 1,25-dihydroxyvitamin $\mathrm{D}(3)$ by human endothelial cells is regulated by inflammatory cytokines: a novel autocrine determinant of vascular cell adhesion. J Am Soc Nephrol 2002;13:621-629.

4 Veloudi P, Jones G, Sharman JE: Effectiveness of vitamin D supplementation for cardiovascular health outcomes. Pulse 2016;4:193-207.

5 Beveridge LA, Struthers AD, Khan F, Jorde R, Scragg R, Macdonald HM, et al: Effect of vitamin D supplementation on blood pressure: a systematic review and meta-analysis incorporating individual patient data. JAMA Intern Med 2015;175:745-754.

6 Upala S, Sanguankeo A, Congrete S, Jaruvongvanich V: Effect of cholecalciferol supplementation on arterial stiffness: a systematic review and meta-analysis. Scand Cardiovasc J 2016;50:230-235.

7 Rodríguez AJ, Scott D, Srikanth V, Ebeling P: Effect of vitamin D supplementation on measures of arterial stiffness: a systematic review and meta-analysis of randomized controlled trials. Clin Endocrinol 2016;84: 645-657.

8 Hussin AM, Ashor AW, Schoenmakers I, Hill T, Mathers JC, Siervo M: Effects of vitamin D supplementation on endothelial function: a systematic review and meta-analysis of randomised clinical trials. Eur J Nutr 2016, Epub ahead of print.

9 Theodoratou E, Tzoulaki I, Zgaga L, Ioannidis JPA: Vitamin D and multiple health outcomes: umbrella review of systematic reviews and meta-analyses of observational studies and randomised trials. BMJ 2014;348:g2035.

10 Ford JA, MacLennan GS, Avenell A, Bolland M, Grey A, Witham M, et al: Cardiovascular disease and vitamin D supplementation: trial analysis, systematic review, and meta-analysis. Am J Clin Nutr 2014;100:746-755.

11 Elamin MB, Abu Elnour NO, Elamin KB, Fatourechi MM, Alkatib AA, Almandoz JP, et al: Vitamin D and cardiovascular outcomes: a systematic review and meta-analysis. J Clin Endocrinol Metab 2011;96:1931-1942.

12 Autier P, Gandini S: Vitamin D supplementation and total mortality: a meta-analysis of randomized controlled trials. Arch Intern Med 2007;167:1730-1737.

13 Giovannucci E: Can vitamin D reduce total mortality? Arch Intern Med 2007;167:1709-1710.

14 Wang L, Manson JE, Song Y, Sesso HD: Systematic review: vitamin D and calcium supplementation in prevention of cardiovascular events. Ann Intern Med 2010;152:315-323.

15 Zheng Y, Zhu J, Zhou M, Cui L, Yao W, Liu Y: Meta-analysis of long-term vitamin D supplementation on overall mortality. PLoS One 2013;8:e82109.

16 Margolis KL, Ray RM, Van Horn L, Manson JE, Allison MA, Black HR, et al: Effect of calcium and vitamin D supplementation on blood pressure: the Women's Health Initiative Randomized Trial. Hypertension 2008;52: 847-855.

17 Daly RM, Nowson CA: Long-term effect of calcium-vitamin D(3) fortified milk on blood pressure and serum lipid concentrations in healthy older men. Eur J Clin Nutr 2009;63:993-1000.

18 Orwoll ES, Oviatt S: Relationship of mineral metabolism and long-term calcium and cholecalciferol supplementation to blood pressure in normotensive men. Am J Clin Nutr 1990;52:717-721. 